\title{
Modeling Alignment Experiment Errors for Improved Computer-Aided Alignment
}

\author{
Yunjong Kim ${ }^{1,2}$, Ho-Soon Yang ${ }^{1,2 *}$, Jae-Bong Song ${ }^{2}$, Sug-Whan Kim ${ }^{3,4}$, and Yun-Woo Lee ${ }^{2}$ \\ ${ }^{I}$ Department of Science of Measurement, University of Science and Technology, Daejeon 305-350, Korea \\ ${ }^{2}$ Center for Space Optics, Korea Research Institute of Standards and Science, Daejeon 305-340, Korea \\ ${ }^{3}$ Space Optics Laboratory, Department of Astronomy, Yonsei University, Seoul 120-749, Korea \\ ${ }^{4}$ Center for Galaxy Evolution Research, Yonsei University, Seoul 120-749, Korea
}

(Received November 6, 2013 : accepted November 18, 2013)

\begin{abstract}
Contrary to the academic interests of other existing studies elsewhere, this study deals with how the alignment algorithms such as sensitivity or Differential Wavefront Sampling (DWS) can be better used under effects from field, compensator positioning and environmental errors unavoidable from the shop-floor alignment work. First, the influences of aforementioned errors to the alignment state estimation was investigated with the algorithms. The environmental error was then found to be the dominant factor influencing the alignment state prediction accuracy. Having understood such relationship between the distorted system wavefront caused by the error sources and the alignment state prediction, we used it for simulated and experimental alignment runs for Infrared Optical System (IROS). The difference between trial alignment runs and experiment was quite close, independent of alignment methods; $6 \mathrm{~nm}$ rms for sensitivity method and $13 \mathrm{~nm}$ rms for DWS. This demonstrates the practical usefulness and importance of the prior error analysis using the alignment algorithms before the actual alignment runs begin. The error analysis methodology, its application to the actual alignment of IROS and their results are described together with their implications.
\end{abstract}

Keywords : Alignment, CAA, Singular value decomposition

OCIS codes : (220.1140) Alignment; (220.4840) Testing

\section{INTRODUCTION}

Over the last few decades or so, a number of computeraided alignment estimation methods have been developed. Figoski et al. [1] used the sensitivity table method for alignment of a wide field, three mirror system. Liu et al. [2] found the best set of compensators by calculating the condition number of the sensitivity matrix combination for the target optical system. In the meantime, Kim et al. [3] reported the merit function regression (MFR) method utilizing an optimization algorithm benefitted from multiple wavefront error (WFE) measurements. Generally, they are used to estimate the misalignment state of the selected compensators by using the WFE measurement from multiple fields. On the other hand, Lee et al. [4] proposed Differential Wavefront Sampling (DWS) using the second derivative terms of astigmatism (i.e. diagonal astigmatism,
Z5) and the single center field WFE measurement along the optical axis.

While their capability has been well documented however, aforementioned methods tend to neglect the combined effects of practical alignment errors onto the alignment state estimation. These include spatial and temporal variations of environmental conditions in WFE measurement, motion accuracies inherent from the use of alignment tools, etc. In earlier studies, researchers addressed the above effects individually and, to lesser extents, for performance verification of their computer-aided alignment algorithms used in alignment of their target optical system [4-7]. However, the actual alignment runs were made in inadequate accuracy range so that their algorithm performances were not investigated in detail. Furthermore, the effects of field positioning inaccuracy has never been taken into account. Hvisc et al. [8] attempted the singular value decomposition

\footnotetext{
*Corresponding author: hsy@kriss.re.kr

Color versions of one or more of the figures in this paper are available online.
} 
(SVD) analysis to understand the aberration modes when aligning the wide field corrector of the Hobby-Eberly Telescope. Their study resulted in a relationship between the optical component position and their aberration effects, while it did not address the alignment effects from the aforementioned error sources.

There are three important error sources involved in the alignment experiment; field, compensator positioning, and environmental errors. The focus of the interferometer can be placed in an unintended position (i.e. field error) or the movement of compensators can have errors due to the imperfection or low resolution of motion stages (i.e. micrometer positioning error). More critically, the environmental conditions such as air turbulence or vibration during the WFE measurement may affect the resulting interferogram, which causes variations in the Zernike coefficients that are key values to computer-aided alignment (i.e. environmental error). These three errors tend to affect the alignment result in different manners, depending on the algorithm to be used. Therefore, it would be of great usefulness, if the alignment effects of these error sources and their relationships with the alignment state estimation algorithms were analyzed before computeraided alignment experiment is commenced in optics shops.

In this paper, we first studied the alignment state estimation performances of two algorithms i.e. the sensitivity method and DWS technique under the presence of the above mentioned error sources. We then applied our findings to both simulated and actual computer-aided alignment runs for an infrared optical system (IROS) [9]. Since the two methods use different number of fields and different Zernike coefficients measured, we checked the usefulness of our findings by comparison of their performance results. In Section 2, we describe the error analysis concept and methodology in details. These are followed by the IROS alignment experiment and results as shown in Section 3. Section 4 is concerned with discussion and implications of the analysis results.

\section{ALIGNMENT EXPERIMENT ERROR MODELS}

\subsection{Target Optical System: IROS}

Figure 1 shows the $f / 2.7$ IROS schematic diagram. It consists of three mirrors (M6, M7, and M8) and four lenses (L1, L2, L3, and L4). The front-end telescope, not shown in the figure, delivers the incident light beam onto a dichroic beam splitter (BS) where it is divided into the electro-optical (EO) and infrared (IR) wavelength bands. The EO band ( $400 \mathrm{~nm} \sim 900 \mathrm{~nm}$ ) is focused onto a CCD detector directly after being reflected from BS, while the IR band $(3 \mu \mathrm{m} \sim 5 \mu \mathrm{m})$ is transmitted through BS and focused onto an IR detector via IROS. The front-end telescope and IROS can be developed separately and assembled together in modular manner. The IROS WFE requirement was to be around $225 \mathrm{~nm}$ rms. This was to

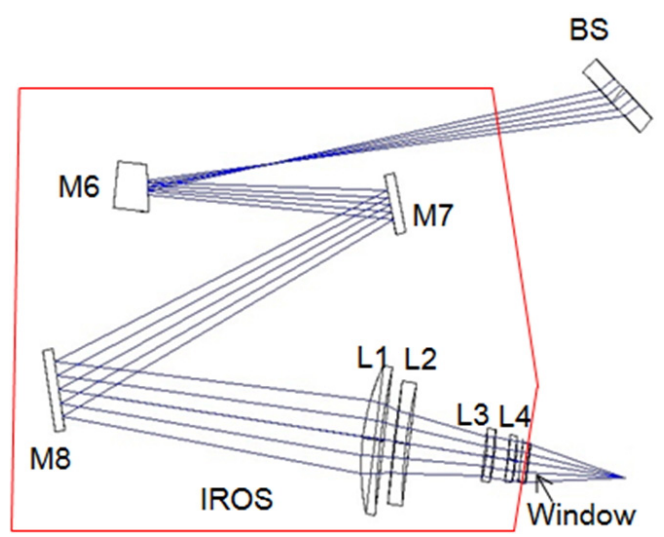

FIG. 1. Schematic diagram of the infrared optical system (IROS).

ensure near-diffraction limited performance at the system level.

\subsection{Environmental Enor}

The WFE measurement with an interferometer is sensitive to air turbulence and instrument vibration while the measurement data is taken. The influences of environmental disturbances to the estimation of the alignment state were investigated as explained below and in Fig. 2.

1. Amount of initial misalignment of each compensator is input. They are generally in sub-millimeter scale. This misalignment is to be estimated and then corrected from using the alignment algorithms.

2. The Zernike coefficients of the misaligned optical system wavefront are computed. The sensitivity method uses 5 Zernike coefficients from 5 measurement fields (on-axis and 4 edge diagonal fields); $0^{\circ}, 45^{\circ}$ astigmatism $(\mathrm{Z} 5, \mathrm{Z6}), \mathrm{x}, \mathrm{y}$ coma $(\mathrm{Z7}, \mathrm{Z8})$ and $3^{\text {rd }}$ order spherical aberration (Z9) coefficients. For the DWS method, the field is fixed at the center along the optical axis and the positions of compensators are deliberately perturbed back and forth to obtain two sets of 5 Zernike coefficients (Z5 Z9).

3. The misaligned system rms WFE (W) can be approximated from the root-sum-square (RSS) of the measured Zernike coefficients (Z5 Z9) obtained from all fields in the sensitivity method and all positions in the DWS method.

4. The environmental error range $\left(\sigma_{1}\right)$ is then set. It is experimentally determined from using the standard deviation (SD) of repeatable WFE measurement in actual alignment practice.

5. The random numbers $\left(\alpha_{\mathrm{i}}\right)$ are generated according to a normal distribution with zero average and $\mathrm{SD}$ of $\sigma_{1}$. These numbers are added to each Zernike coefficient to make an erroneous Zernike coefficient $\left(Z_{i}+\alpha_{i}\right)$. This is to simulate the random effects of environmental variation such as instrument vibration or air turbulence. We then calculate the RSS of the erroneous 
Zernike coefficients $\left(\mathrm{W}_{\mathrm{E}}\right)$.

6. If the difference between $\mathrm{W}$ and $\mathrm{W}_{\mathrm{E}}$ is larger than $\sigma_{1}$, other random numbers are generated to produce a new set of erroneous Zernike coefficients in step 5. This process is repeated until the criterion is met as in step 6 in Fig. 2.

7. Employing alignment algorithms, the amounts of current misalignments for each compensator is derived. The final rms WFE is then calculated after compensating for the estimated initial misalignments.

8. The process step $5 \sim 7$ are repeated 100 times to obtain the averaged rms WFE and its standard deviation.

Figure 3 shows the analysis results at the four edge fields. The initial misalignments of compensators were set to $0.5 \mathrm{~mm}$. The environmental error used varies from $0.1 \mathrm{~nm}$ to $30 \mathrm{~nm} \mathrm{rms}$. $0.1 \mathrm{~nm}$ was used to simulate the best

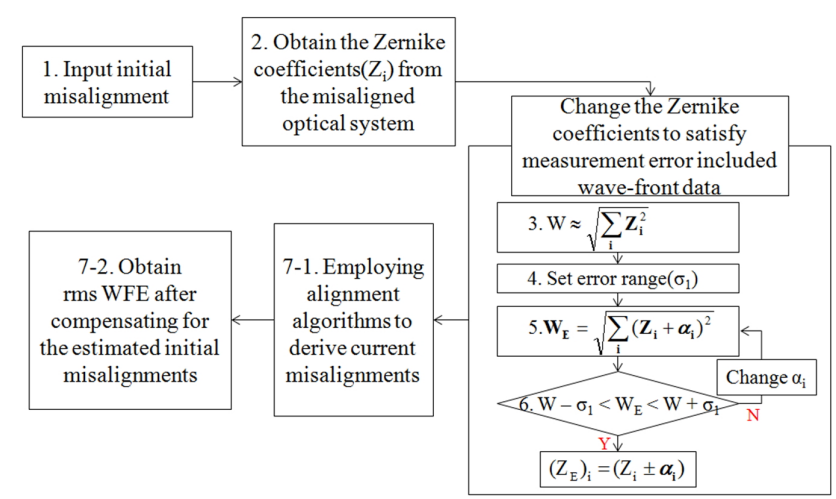

FIG. 2. Flow chart for simulating the influence of environmental disturbances to the estimation of the alignment state.

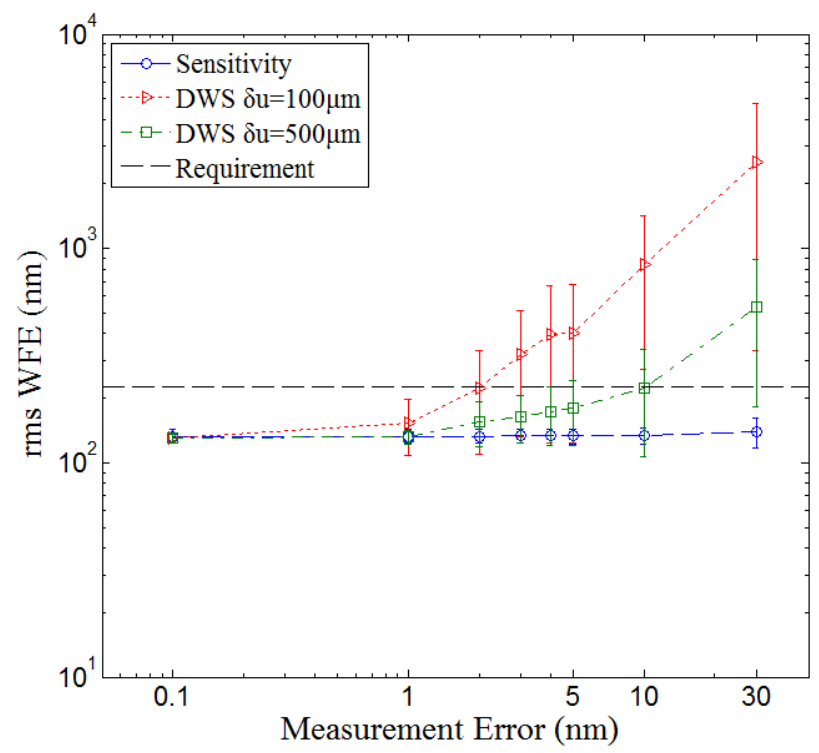

FIG. 3. Simulation results of sensitivity and DWS methods related to the environmental error. Black dashed line is the requirement and $\delta \mathrm{u}$ is the range of deliberate perturbation. measurement accuracy of a commercial interferometer, while $30 \mathrm{~nm}$ was to represent the measurement under the harsh environmental conditions. The total 100 iterative computations were carried out for each environmental error input.

For the DWS method, two different perturbations $(\delta \mathrm{u})$ of $100 \mu \mathrm{m}$ and $500 \mu \mathrm{m}$ for each compensator were used. Initially, the $500 \mu \mathrm{m}$ perturbation case was analyzed to set the reference response, even though it generated WFE too large to be measured with a commercial Fizeau type interferometer. Figure 3 shows remarkably stable results within the requirement from the sensitivity method, while the DWS method tends to increase rms WFE significantly beyond the requirement as the measurement error increases. We believe that the higher robustness of the sensitivity method than for DWS comes from the average-out effects of random environmental errors by using more parameters; the sensitivity method utilizes 25 parameters (i.e. 5 Zernike coefficients at 5 different fields), whereas there are only 10 parameters used for DWS method (i.e. 1 Zernike coefficients, which is Z5, at 10 different positions - 5 compensators per deliberate perturbation at single field).

\subsection{Measurement Field Error}

As the sensitivity method uses multiple field WFE measurements while DWS uses the single field, the measurement field error can be further amplified for the sensitivity method than for DWS. Figure 4(a) shows the concept of multiple field WFE measurement. A reference iron ball is placed at the measurement focal plane and the distance variation between the interferometer and the focal plane is continuously adjusted. In this arrangement, the field position error in the optical axis (Z) direction plays an insignificant role during the measurement. We can then consider $\mathrm{x}$ and $\mathrm{y}$ axis position errors as in Fig. 4(b). FX and $\mathrm{FY}$ are field directions along $\mathrm{x}$ and $\mathrm{y}$ axis. $\mathrm{F}_{\mathrm{i}}$ is the ideal position, $\mathrm{F}_{\mathrm{E}}$ the actual position with the error included, $\sigma_{2}$ the maximum field error in radius and $a\left(\alpha_{2 i-1}\right.$ and $\alpha_{2 i}$ means odd and even numbers) the error at each axis. After setting up the interferometer position, the iron ball is removed and the IROS WFE is measured. In this manner,

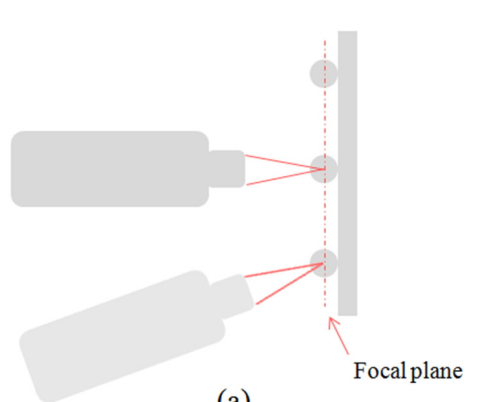

(a)

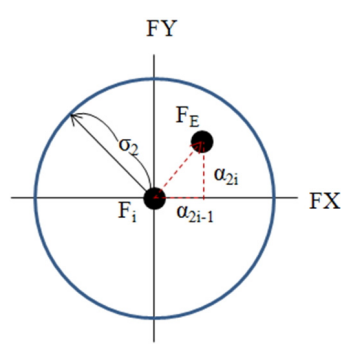

(b)
FIG. 4. (a) Concept of multiple fields WFE measurement with iron ball (b) Schematic diagram of the field error at i-th field with given error range $\left(\sigma_{2}\right)$. 
we can measure and analyze the field error for the sensitivity method.

For analysis of field error in the sensitivity method, the following steps were applied as shown in Fig. 5.

1. The analysis step 1 is as same as in the environmental error case already mentioned. The amount of initial misalignment is given to each compensator and it needs to be computed and corrected.

2. The total 10 random field errors with given range $\left(\sigma_{2}\right)$ are generated and added to each normalized field to represent field error; $\left(\left(0 \pm \alpha_{1}, 0 \pm \alpha_{2}\right),\left(1 \pm \alpha_{3}, 1 \pm \alpha_{4}\right)\right.$, $\left.\left(1 \pm \alpha_{5},-1 \pm \alpha_{6}\right),\left(-1 \pm \alpha_{7}, 1 \pm \alpha_{8}\right),\left(-1 \pm \alpha_{9},-1 \pm \alpha_{10}\right)\right)$. The $\mathrm{z}$-axis error is not considered, as it is calibrated with the iron ball at the focal plane.

3. We obtain $0^{\circ}$ and $45^{\circ}$ astigmatism $(Z 5, Z 6), x$ and $y$ coma $(\mathrm{Z7}, \mathrm{Z8})$ and $3^{\text {rd }}$ order spherical aberration (Z9) coefficients from 5 measurement fields (on-axis and 4 edge diagonal fields) inclusive of the field position errors.

4. The alignment algorithm is then used to estimate the

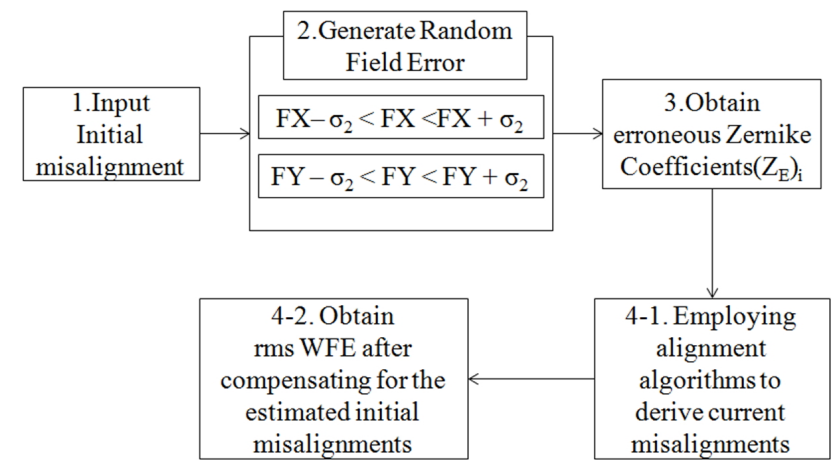

FIG. 5. Flow chart for simulating the influence of field error to the estimation of the alignment state.

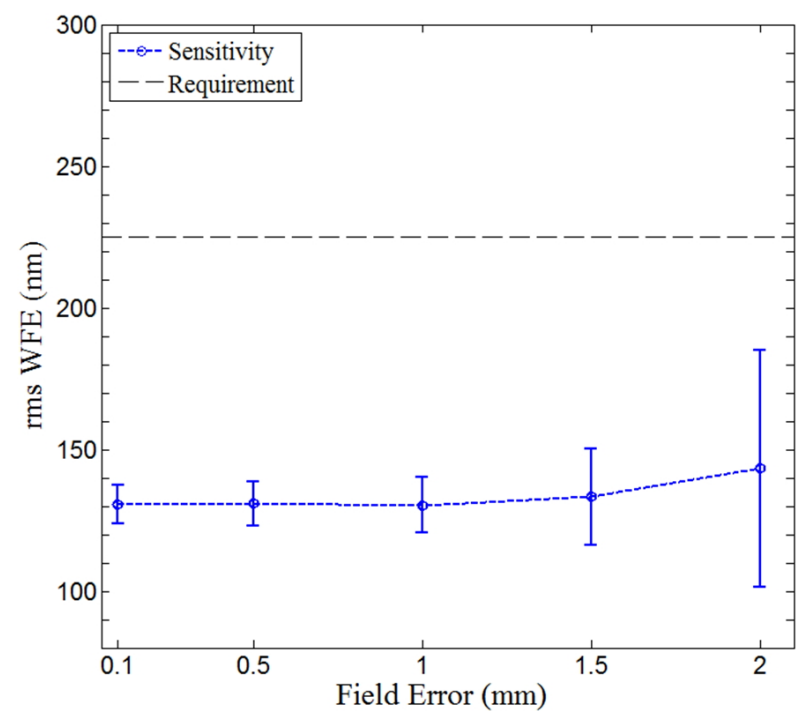

FIG. 6. Simulated rms WFE versues field error for sensitivity method. Black dashed line is the requirement. current misalignment for each compensator. The final rms WFE is then obtained after compensating for the estimated current misalignments.

5. The process steps from 2 to 4 are repeated to derive the average rms WFE and its SD.

Figure 6 shows the analysis results, showing the mean WFE and SD bar from 100 iterative simulations at 5 fields (4 edge fields and on-axis field). The aligned WFE is located within the requirement for all fields over the field error range from 100 microns to $2 \mathrm{~mm}$. This implies that the sensitivity method is very well immune to the field position error in wavefront measurement.

\subsection{Micrometer Positioning Error}

For the case of the sensitivity method, the positioning error does not occur, as the compensators are stationary during the WFE measurement. On the other hand, the positioning error of compensator stages affects the alignment estimation performance of the DWS method as it requires for the deliberate perturbation of compensator location along the mount axis, in order to obtain the gradient of $0^{\circ}$ astigmatism (Z5). The concept of the positioning error model is depicted in Fig. 7. Here the decenter of L3 and L4 in $\mathrm{X}$ and $\mathrm{Y}$, and their lateral shift along the light path are of main interests to the analysis model. Their intended motion $(\mathrm{Su})$ and its unexpected error $\left(\sigma_{3}\right)$ taking place during the alignment action are drawn in Fig 7.

The model analysis steps follow the procedure listed below and as shown in Fig. 8 .

1. The analysis step 1 is as same as in the environmental error case already mentioned. The amount of initial misalignment is given to each compensator and it needs to be computed and corrected.

2. The random positioning error within the range $\left(\sigma_{3}\right)$ is generated and input to the deliberately perturbation of the compensator back $(-\delta u)$ and forth $(+\delta u)$. This is to represent the position accuracy of the compensator mount in alignment action.

3. We then obtain the erroneous $0^{\circ}$ astigmatism (Z5)

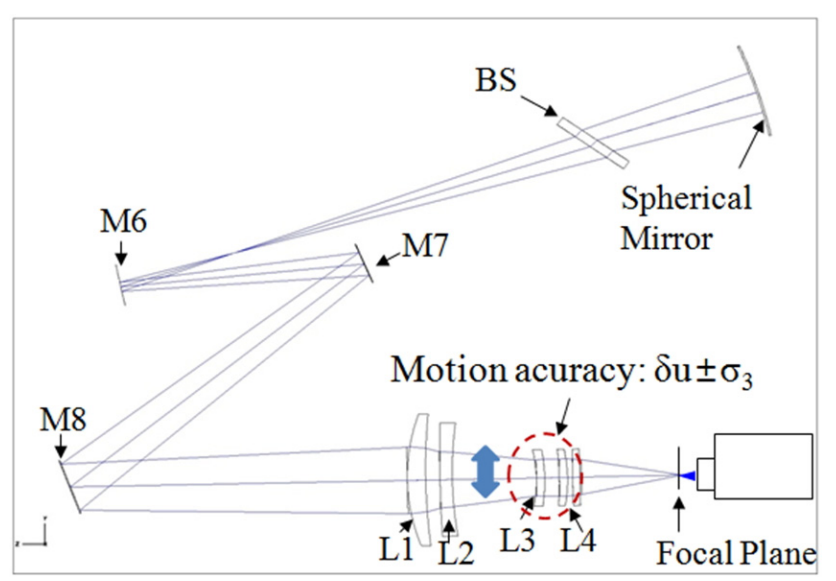

FIG. 7. Schematic diagram of the stage position error. 
coefficients from the on-axis field at back $\left(\mathrm{M}_{\mathrm{E}(-)}\right)$ and forth $\left(\mathrm{M}_{\mathrm{E}(+)}\right)$ positions.

4. The gradients of $\mathrm{Z} 5$ are computed for each compensator movement. $\mathrm{Z5_{(+) }}$ is obtained from the forward $\left(\mathrm{M}_{\mathrm{E}(+)}\right)$ perturbation positions and $\mathrm{Z5_{(-) }}$ from backward $\left(\mathrm{M}_{\mathrm{E}(-)}\right)$ perturbation positions. For the calculation, the deliberate perturbation range is fixed to $2 \delta \mathrm{u}$.

5. The alignment algorithm is used to estimate the current misalignments of each compensator. The final rms WFE is then obtained after compensating for the estimated current misalignments.

6. The process steps from 2 to 5 are repeated to obtain the average final rms WFE and its SD.

Figure 9 shows the model analysis results for two perturbation cases of $100 \mu \mathrm{m}$ and $500 \mu \mathrm{m}$. Each point in the graph is the mean WFE and SD bar derived from 100 iterative computations only at the on-axis field. The

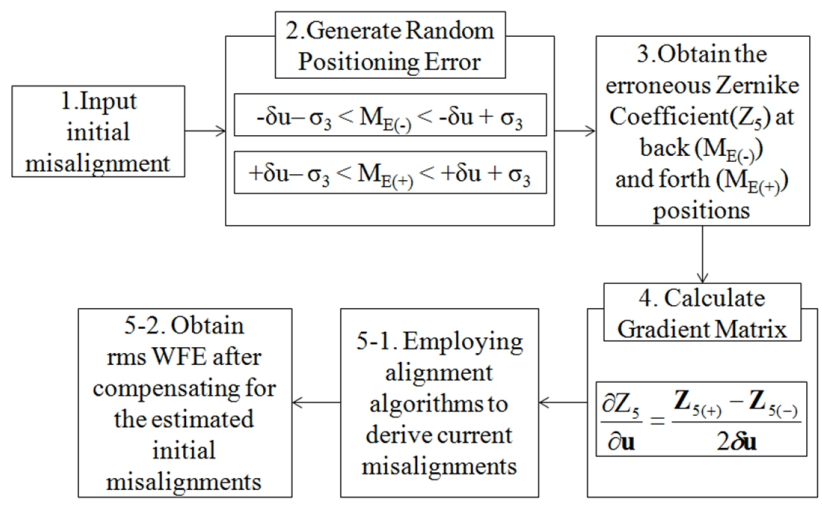

FIG. 8. Flow chart for simulating the influence of micrometer positioning error to the estimation of the alignment state.

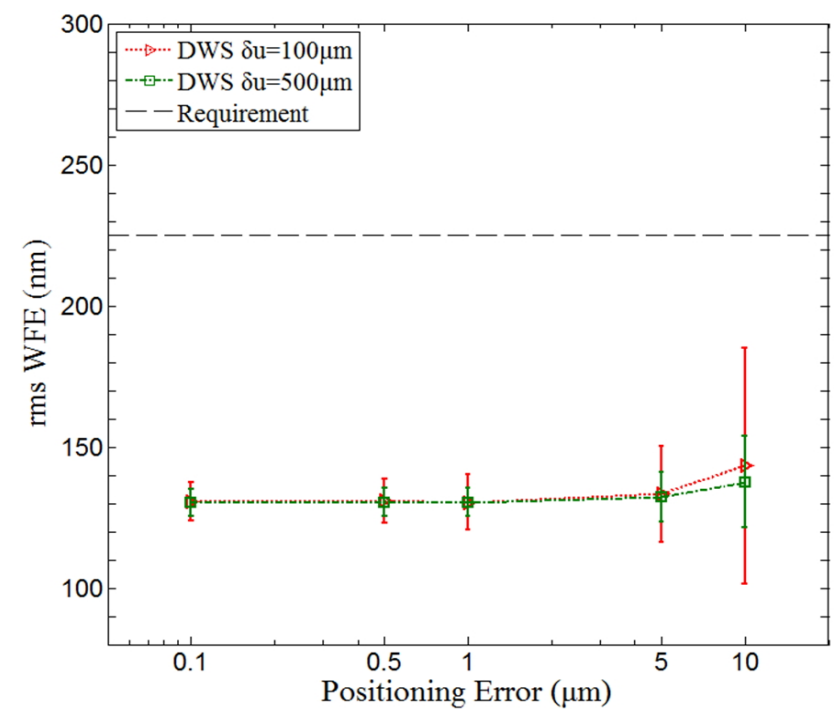

FIG. 9. Micrometer positioning error model applied to DWS method. Black dashed line is the requirement and $\delta \mathrm{u}$ is the range of deliberate perturbation. resulting rms WFE remains stationary over the positioning error range from 0.1 micron to about 5 microns and it increases marginally between 5 microns to 10 microns in range. Nevertheless, the trend is clear that it remains well below the requirement. This implies that the DWS method is practically insensitive to the micrometer positioning error when aligning IROS. In summary, we gathered convincing evidence that, for the actual IROS alignment run, we do not need to be concerned about the field error if the sensitivity method was to be used and the positioning error if the DWS method was to be applied.

\section{ALIGNMENT EXPERIMENTS}

Trial alignment runs were made for IROS with the sensitivity and DWS methods. Figure 10(a) shows the schematic diagram of the alignment set-up and Fig. 10(b) is a photograph of the actual experiment configuration. The IR interferometer sends a spherical wave of $f / 2.5$ to the IROS focal plane. It passes through the transparent window and lens group from L4 to L1. The IR beam is reflected from M8 toward M6. As explained in Section 1, in order to simulate the front-end optics, a parallel plate and a spherical mirror of $100 \mathrm{~mm}$ in diameter and of $529 \mathrm{~mm}$ in curvature of radius are added; the parallel plate acts as a BS of front-end optics and a spherical mirror reflects the spherical wave generated by IROS back to the interferometer.

A Fizeau type IR interferometer operating at $3.39 \mu \mathrm{m}$ in the wavelength of the He-Ne laser was used for the wavefront measurement. As expressed already, the initial sensitivity analysis defined the compensators for alignment as L3 decenter (x,y), L4 decenter $(\mathrm{x}, \mathrm{y})$ and L4 despace (see Figure 1) [10]. The tolerances were set to $0.05 \mathrm{~mm}$ for all the compensators. The main compensator L3 and L4 were mounted on a commercial two-axis linear stage with $10 \mu \mathrm{m}$ in micrometer resolution. The IROS optical design defines $124 \mathrm{~nm}$ in rms WFE for the center field and $225 \mathrm{~nm}$ for the whole field target performance. The IROS is designed to operate $4200 \mathrm{~nm}$ in wavelength and, with the above mentioned WFE target, it is expected to show a near diffraction limited performance.

100 WFE measurements were made at each field and the resulting WFE data were averaged to understand the environmental error influence to the system wavefront. The measurements were made over all 5 fields and the results show $2 \mathrm{~nm} \mathrm{rms}$ in SD. This leads to, according to Fig. 3, the IROS alignment state influence of $132 \mathrm{~nm}$ would be arising from the sensitivity method and of $223 \mathrm{~nm}$ with $115 \mathrm{~nm}$ in SD from the DWS method under the deliberate perturbation of $100 \mu \mathrm{m}$. It would be further aggravated if we take the surface error of $30 \mathrm{~nm} \mathrm{rms}$ in average for total 14 surfaces into consideration. In this case, the alignment state would worsen to $174 \mathrm{~nm}$ in RSS for the sensitivity method and $249 \mathrm{~nm}$ for the DWS method. Therefore, it was predicted that the application of 


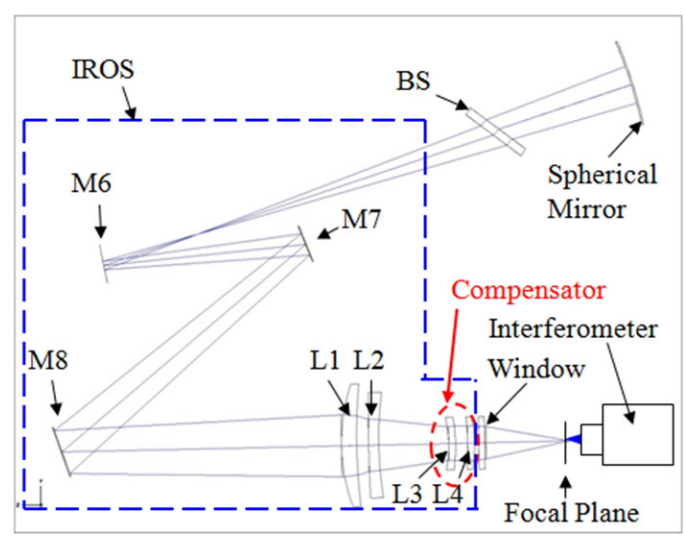

(a)

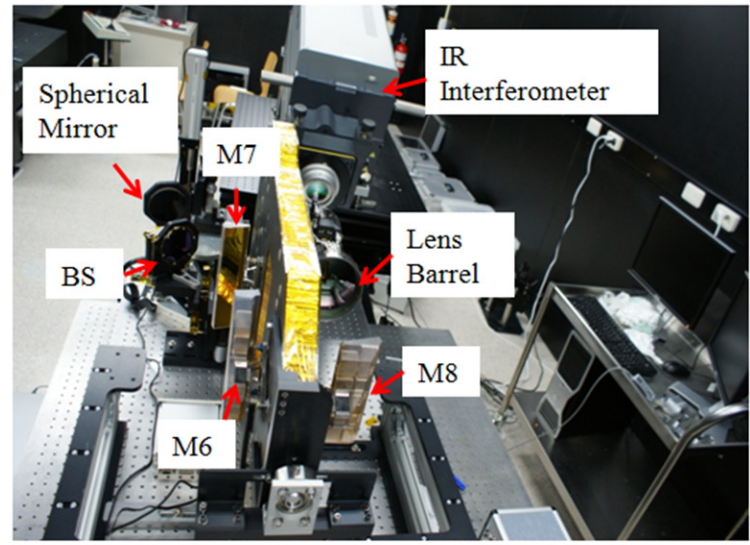

(b)

FIG. 10. (a) Schematic diagram of the alignment configuration and (b) Experimental setup.

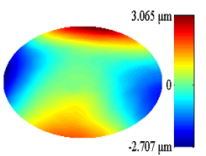

(a) $(-1,1)$ field

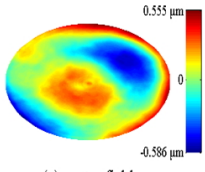

(c) center field

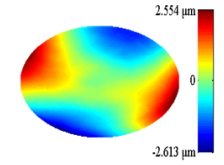

(d) (-1,-1) field

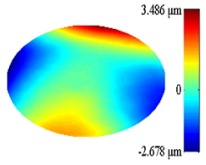

(b) $(1,1)$ field

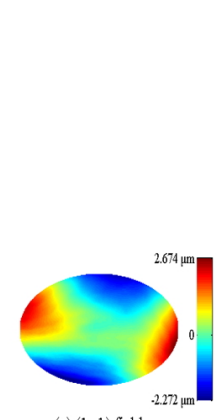

(e) $(1,-1)$ field

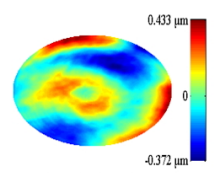

(a) $(-1,1)$ field

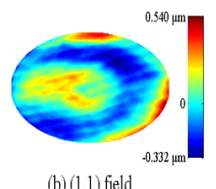

(b) $(1,1)$ field

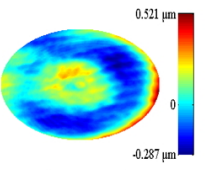

(c) center field

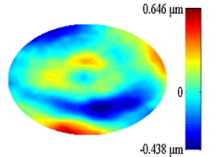

(d) $(-1,-1)$ field

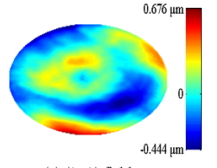

(e) $(1,-1)$ field
FIG. 11. WFEs measured straight after the IROS integration, but before the fine alignment using alignment algorithms: (a) 995 $\mathrm{nm}$, (b) $1001 \mathrm{~nm}$, (c) $216 \mathrm{~nm}$, (d) $1004 \mathrm{~nm}$, and (e) $966 \mathrm{~nm}$. Piston, tip-tilt, and defocus term removed.

sensitivity method to the IROS alignment would satisfy $225 \mathrm{~nm}$ in the target WFE requirement, while the DWS method would not produce such successful results.

Having understood the aforementioned environmental influences to the alignment state estimation, we measured rms WFE at the center and 4 edge fields before the commencement of the IROS fine alignment. As shown in Fig. 11, the results vary from $966 \mathrm{~nm}$ to $1004 \mathrm{~nm}$ over 4 edge fields and $216 \mathrm{~nm}$ at the center. These are clearly beyond the requirement of $225 \mathrm{~nm} \mathrm{rms}$. From the measurement data, the Zernike coefficient set was extracted for the sensitivity method while, for the DWS method, Z5 (0 degree astigmatism) was derived at the center field with $100 \mu \mathrm{m}$ of perturbation in both directions of compensators. This amount of perturbation was nearly the maximum limit of the practical WFE measurement with a commercial Fizeau type IR interferometer for IROS.
FIG. 12. WFE measurement results after alignment runs with sensitivity method: (a) $157 \mathrm{~nm}$, (b) $157 \mathrm{~nm}$, (c) $130 \mathrm{~nm}$, (d) 171 $\mathrm{nm}$, and (e) $188 \mathrm{~nm}$. Piston, tip-tilt and defocus errors removed.

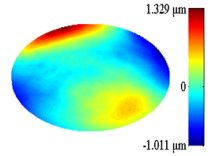

(a) $(-1,1)$ field

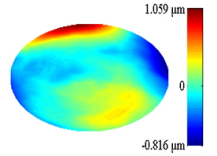

(b) $(1,1)$ field

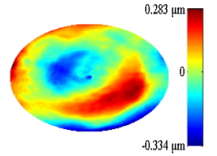

(c) center field

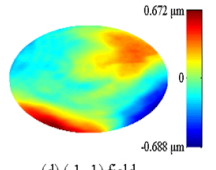

(d) $(-1,-1)$ field

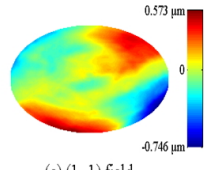

(e) $(1,-1)$ field
FIG. 13. WFE measurement results after alignment runs using DWS method: (a) $359 \mathrm{~nm}$, (b) $275 \mathrm{~nm}$ (c) $116 \mathrm{~nm}$ (d) $209 \mathrm{~nm}$ (e) $208 \mathrm{~nm}$. Piston, tip-tilt and defocus error terms removed. 
Figure 12 shows the WFE measurement results after the alignment run using the sensitivity method. The resulting WFE distribution varies from $157 \mathrm{~nm}$ to $188 \mathrm{~nm}$ for the off-axis fields (168 nm in average) and they are quite close to $174 \mathrm{~nm}$ that is the theoretical expectation. The alignment results with DWS method are shown in Fig. 13, ranging from $208 \mathrm{~nm}$ to $359 \mathrm{~nm}$ for the off-axis fields (262 $\mathrm{nm}$ in average) and $116 \mathrm{~nm}$ for the on-axis center field. These values also fall close to our expectation, 249 $\mathrm{nm}$, even though the application of DWS method to the IROS alignment would produce less successful results across all fields. Specially, the WFEs of $359 \mathrm{~nm}$ at $(-1,1)$ field and $275 \mathrm{~nm}$ at $(1,1)$ field do not meet the requirement of $225 \mathrm{~nm}$, whereas the other field shows marginally close to satisfaction. In general, the above mentioned experimental results demonstrate that the prior analysis of practical error sources in alignment can be an effective tool for choosing the proper alignment algorithm and for efficient alignment process.

\section{DISCUSSION}

Figure 14 shows both predicted and experimented rms WFE data of IROA just after the initial integration and after the fine alignment. The measured environmental wavefront error of $2 \mathrm{~nm} \mathrm{rms}$ and the averaged surface figure error of $30 \mathrm{~nm} \mathrm{rms} \mathrm{WFE} \mathrm{for} 14$ surfaces in total were added to form the data shown in the figure. The initial wavefront error is the averaged value of all the wavefront error data appeared in Fig. 11, while those of "Experiment" and "Simulation" are from the data depicted in Fig. 12, Fig. 13. and Fig. 3.

First, we note that the simulated average rms WFE is $174 \mathrm{~nm}$ and it is very close to $168 \mathrm{~nm}$ from the experiment. And their field imbalances are $10 \mathrm{~nm}$ in simulation and 14 $\mathrm{nm}$ from the measurement respectively. On the other hand, the average rms WFE from the DWS simulation turns out to be $249 \mathrm{~nm}$ but $262 \mathrm{~nm}$ in experiment. Their field imbalances are $112 \mathrm{~nm}$ in simulation and $71 \mathrm{~nm}$ in experiment respectively. The close proximity of predicted and experimental WFE tells us that all the influences from those practical alignment error sources have been well accounted for in both simulation and experiment analysis of the alignment state. We also note that the field error effects to the sensitivity method performance and the positioning error effects to the DWS method performance are negligible as predicted in section 2 . However, the error analysis can suggest how the DWS method can be better used; for example, if the environmental error can be reduced to less than $1 \mathrm{~nm} \mathrm{rms}$ or the optical system is capable of dealing with $400 \mu \mathrm{m}$ or larger perturbation, then the IROS can be aligned to within the requirement by using the DWS method. To those extents, the error analysis method presented in this study is an efficient tool to increase the WFE estimation performance of the

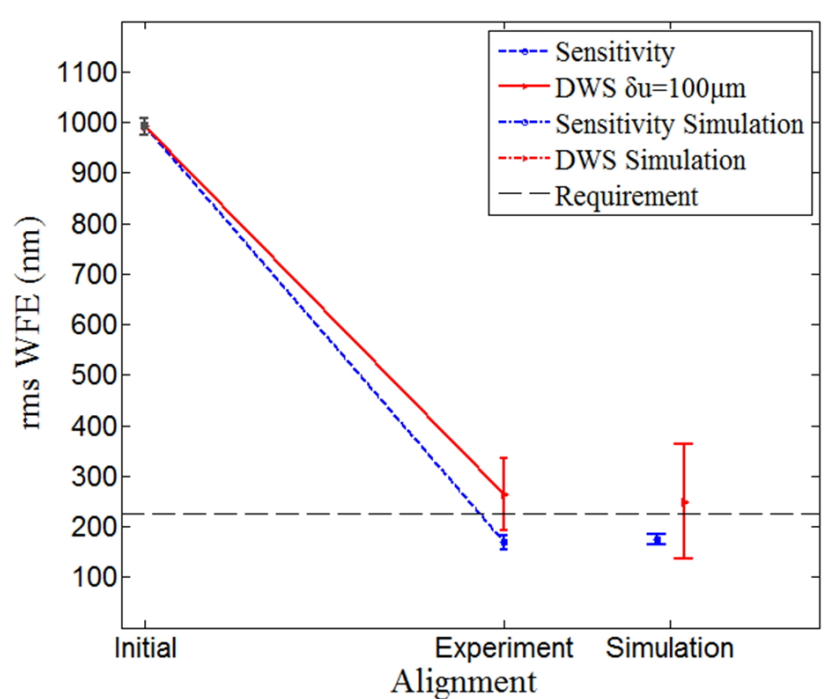

FIG. 14. Evolution of rms WFE in simulated and experimental alignments of IROS. The simulation result is obtained with the measured environmental and surface figure errors mentioned above. Black dashed line is the requirement and $\delta u$ the deliberate perturbation applied to DWS.

alignment algorithm and therefore the process efficiency of actual alignment run.

\section{CONCLUSION}

While environmental, measurement field and micrometer positioning errors play important roles in decreasing the process efficiency of the computer-aided alignment, earlier studies [1-7] gave less academic attention to these very subjects. On the contrary, this study addresses detailed modeling and analysis of such error sources influencing the alignment estimation performance for actual alignment run. The analysis results show that the environmental error plays the most significant role in degrading the alignment process efficiency, whereas the influences from the field and positioning error can be negligible depending on the choice of alignment algorithm used.

We applied these findings to the application of sensitivity and DWS algorithms for alignment of IROS both in simulation and in experiment. The results show that the sensitivity method gave the aligned WFE thus satisfying the requirement, while the DWS method was less successful. This demonstrates that, with prior error model analysis of the target optical system using the alignment algorithms, a proper alignment method can be selected depending on what plays the role of the primary alignment error source, even before the actual alignment run commences. We believe that the work and findings presented in this study would contribute greatly to the increasing process efficiency for actual alignment run during the final integration and testing phase for high performance optical systems. 


\section{ACKNOWLEDGMENT}

This work was mainly supported by Korea Research Council of Fundamental Science \& Technology - Grant funded by the Korean Government (KRCF-2012-CAP-1345194477).

The manuscript preparation was supported, in part, by NRF grant 2012-0006632 and the SRC program 2010-0027910 of Center for Galaxy Evolution Research.

\section{REFERENCES}

1. J. W. Figoski, T. E. Shrode, and G. F. Moore, "Computeraided alignment of a wide field, three-mirror, unobscured, high-resolution sensor," Proc. SPIE 1049, 166-177 (1989)

2. J. Liu, F. Long, and W. Zhang, "Study on computer-aided alignment method," Proc. SPIE 5638, 674-681 (2005).

3. S. Kim, H.-S. Yang, Y.-W. Lee, and S.-W. Kim, "Merit function regression method for efficient alignment control of two-mirror optical systems," Opt. Express 15, 5059-5068 (2007).

4. H. Lee, G. B. Dalton, I. A. J. Tosh, and S.-W. Kim, "Computer-guided alignment II: Optical system alignment using differential wavefront sampling," Opt. Express 15,
15424-15437 (2007).

5. B. F. C. de Albuquerque, R. V. F. Lopes, H. K. Kuga, E. G. de Carvalho, L. C. N. Scaduto, and M. A. Stefani, "Misalignment parameters estimation in refractive optical systems," Proc. SPIE 7068, 70680P (2008).

6. D. C. Redding, N. Sigrist, J. Z. Lou, Y. Zhang, P. D. Atcheson, D. S. Acton, and W. L. Hayden, "Optical state estimation using wavefront data," Proc. SPIE 5523, 212-224 (2004).

7. N. Sigrist, D. C. Redding, J. Z. Lou, Y. Zhang, and S. Basinger, "Optical system alignment via optical state estimation using wavefront measurements," Proc. SPIE 5965, 596524 (2005).

8. A. M. Hvisc and J. H. Burge, "Alignment analysis of four-mirror spherical aberration correctors," Proc. SPIE 7018, 701819 (2008).

9. H.-S. Yang, H. Kihm, I. K. Moon, G.-J. Jung, S.-C. Choi, K.-J. Lee, H.-Y. Hwang, S.-W. Kim, and Y.-W. Lee, "Three-shell-based lens barrel for the effective athermalization of an IR optical system," Appl. Opt. 50, 6206-6213 (2011).

10. H.-S. Yang, H. Kihm, I. K. Moon, J.-B. Song, and Y.-W. Lee, "Stand-alone assembly of IR camera," 61st IAC 10, BI.3.8 (2010). 